\title{
The implementation and quantification of the Vallejos and McKinnon re-entry methodology
}

\author{
IG Morkel Australian Centre for Geomechanics and The University of Western Australia, Australia
}

P Rossi-Rivera The University of Western Australia, Australia

\begin{abstract}
The occurrence of seismicity in high stress hard rock mines poses a challenge to geotechnical engineers and mine management around the world. Only a few practical options are available when the mitigation of seismic risk is considered. One of the most widely used options is the implementation of a re-entry protocol. These protocols are useful at limiting personnel exposure to elevated seismic hazard associated with the occurrence of a firing. There are several methodologies available for determining an appropriate re-entry time. The success rate of these methods varies between sites.

Recent work by Vallejos and McKinnon (2010) suggest a new approach to the re-entry problem. They provide methodology that could be implemented on any mine site with a seismic system. The method evaluates the current response in terms of the statistical properties of the rock mass based on historic responses. Discussions on the practical implementation of the method on a site-wide basis were limited, and did not provide an indication of what could quantitatively be expected from the method.

The Vallejos and McKinnon method could be automated and practically implemented on most mine sites with a comprehensive seismic data record. It was shown the methodology, in some cases, may be an improvement on the widely used 'blanket' rule implemented on many mine sites.
\end{abstract}

Keywords: seismic re-entry, seismic risk, mine seismology

\section{Introduction}

Potvin (2009) argues that an engineer only has a few options available for reducing the risk associated with seismicity. These options include dynamic support design, mine layouts to minimise stress concentrations, and limiting exposure of personnel to the working face. In instances where this is not possible, exposure is controlled in other ways. For high seismic hazard scenarios this is achieved through the use of re-entry protocols. It is well established that after blasting and large events there is an increase in seismic activity (Woodward et al. 2017). The behaviour of seismicity after these blasts and large events can be described by the Modified Omori Law (MOL) as defined by (Utsu 1961). Although the MOL was initially used in tectonic seismology, it is just as effective for mining induced seismicity (Woodward et al. 2017).

There is a need for understanding and determining a reasonable and defensible re-entry time period after each blast. An accurate method would maximise the safety of personnel and limit the loss of equipment, whilst limiting the impact on production. Although several re-entry techniques are available they all rely, to some extent, on the 'experience' or 'expertise' of the engineer on site. Unfortunately, this experience comes at the cost of historical bad judgements (Mendecki 2008) and, when conditions change, that expertise is not readily transferred. For an in-depth discussion refer to Hudyma (2008), Mendecki (2008), Vallejos and McKinnon (2010).

Vallejos and McKinnon (2010) proposed a methodology aiming to limit the effect of the human experience on the interpretation of the seismic sequences. Their method (V\&M method), would allow the onsite engineer to compare the current response to the rock mass statistics based on the historical responses. They proposed three protocols that would indicate when conditions are favourable for re-entry. This paper uses this relatively new V\&M method and applies it to two seismically active underground mines. The 
practical systematic implementation of the method and the challenges for doing so, is described. The extent of 'favourable', as described by this method is also quantified. A 'blanket' rule is applied to the data to provide a base line for the comparison of the performance of the V\&M method.

\section{Systematic implementation of the method}

Vallejos and McKinnon (2010) investigated 340 different seismic sequences from nine different mines with the aim of defining a methodology to determine when conditions are favourable for re-entry. The study states that the single most important characteristic of mining induced seismicity, relevant to the development of re-entry protocols, is that following large seismic events or blasts there is a short-term increase in levels of seismic activity that, over time, decays to background levels. This decay can adequately be described by the MOL, developed from observed decay rates of large earthquake aftershocks.

This section discusses how the V\&M method is used for re-entry and how this method was automated for use on mine sites. To ensure clear distinction between the two methods, the 'V\&M method' will refer to the methodology proposed by Vallejos and McKinnon (2010). The automated version for the V\&M method will be called the 'automated V\&M method'. For clarity sake, the discussion of the proposed methodology and the systematic implementation is separated in following discussions.

To enable its use on a routine basis, the automated V\&M method was implemented into an software app (Woodward et al. 2015) available through the mXrap software (Harris \& Wesseloo 2015). To enable a systematic implementation the methodology needed to be codified. As was shown in the following sections, the codification of the method still conforms to the ideas of the V\&M method.

\subsection{Modified Omori Law}

The MOL is an empirically derived model for aftershock decay, used to analyse the properties of aftershock sequences produced by seismic events. Seismic responses within this context, generally, are satisfactorily described by the MOL with the following equation (Utsu 1961):

$$
\mathrm{n}(\mathrm{t})=\frac{\mathrm{K}}{(\mathrm{c}+\mathrm{t})^{\mathrm{p}}}
$$

where:

$$
\begin{aligned}
& \mathrm{n}(\mathrm{t})=\text { rate of occurrence of aftershocks as a function of the time t elapsed since the main event. } \\
& \mathrm{K} \quad=\text { productivity parameter related to the total number of events in the sequence. } \\
& \mathrm{c} \quad=\text { offset time constant. } \\
& \mathrm{p} \quad=\text { parameter related to the speed of decay. }
\end{aligned}
$$

In order to define a methodology for the development of re-entry protocols, Vallejos and McKinnon (2010) assumed the seismic response of the rock mass after blasting, can be described by the parameters determined from the MOL. It is the distribution of these MOL parameters, which forms the basis of the Monte Carlo simulation used to describe the responses statistically. Vallejos and McKinnon (2010) suggested the use of three protocols to determine when conditions are favourable for re-entry. The following sections will discuss these three protocols and how they were implemented for the two sites under discussion.

\subsection{Seismic envelopes (1st protocol)}

\subsubsection{V\&M method}

The first protocol in the V\&M method is the calculation and use of seismic envelopes to evaluate a current response against the statistics of the historical responses. The envelopes are determined by a Monte Carlo simulation based on the MOL parameters. Figure 1 shows the seismic envelopes, with two different 
responses (blue and red lines). A response in this context refers to the seismic activity related to the trigger (specifically blasts). Each seismic envelope describes the upper percentage value for the responses; that is, for the 90 th percentile line, only $10 \%$ of the responses have exceeded this line historically. Conditions were favourable when a response crosses the envelopes in a sub-horizontal fashion. In Figure 1, the 2nd response (red line) is moving through the envelopes in a sub-vertical direction and, therefore, re-entry is not favourable. On the other hand, the 1st response (blue line) moves in a sub-horizontal direction and, hence, indicates that conditions are favourable for re-entry.

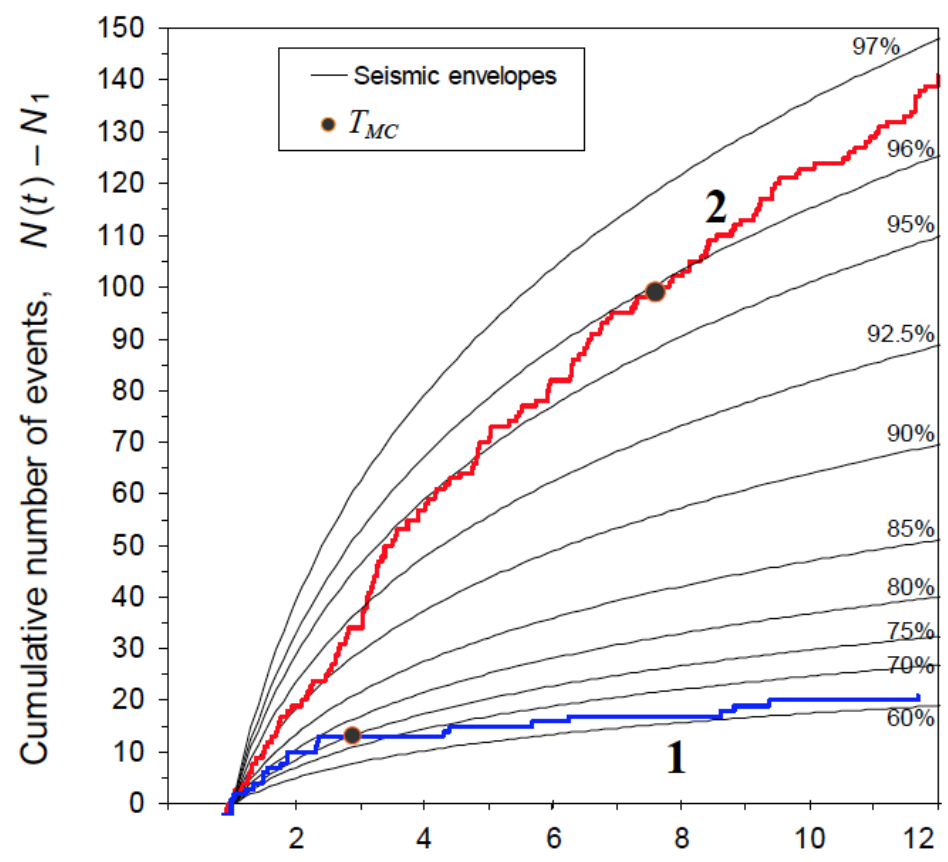

Figure 1 Illustration of seismic envelopes for two seismic paths (Vallejos \& McKinnon 2010)

\subsubsection{Automated V\&M method}

Two challenges were faced during the automation of this protocol. The first, the methodology does not state how much sub-horizontal movement is required to indicate favourable conditions. This, in the V\&M method, is taken to occur when the response crosses through an envelope. The plotted envelope lines in the different cases presented by Vallejos and McKinnon (2010) were not consistent and it appears as though the envelopes were adjusted subjectively to aid the interpretation. To automate and ensure objectivity, the responses were visually expressed in the envelope space; that is, the response is expressed as the percentile value of the Monte Carlo simulation. Such an approach would eliminate the visual ambiguity found in Figure 1. Figure 2 shows the two different ways to view the seismic response. Figure 2(a) indicates the response with the envelopes as proposed in the V\&M method, and in Figure 2(b) the same response is shown in terms of the percentiles as proposed by the automated V\&M method. It is clear that the second visualisation is better at showing the relative movement of the response with regards the envelopes. At four hours after the blast there is an increase in the response, which is clearly seen in Figure 2(b), but not so clearly in Figure 2(a). Sub-horizontal movement in Figure 2(b) is when the response trends downwards. If the response follows an envelope it stays flat and when it is moving sub-vertically it trends upwards. This way of expressing the response gives one the option to implement a threshold value. When a response has moved through the threshold value, it indicates conditions are favourable for re-entry. Sub-horizontal movement for each response is defined as the difference in the maximum percentile value and the current percentile value. 


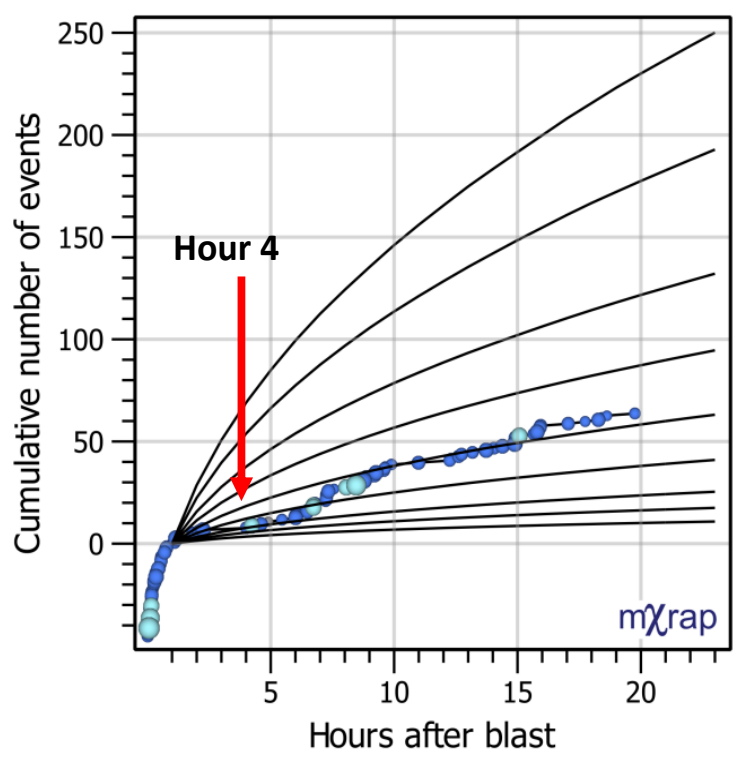

(a)

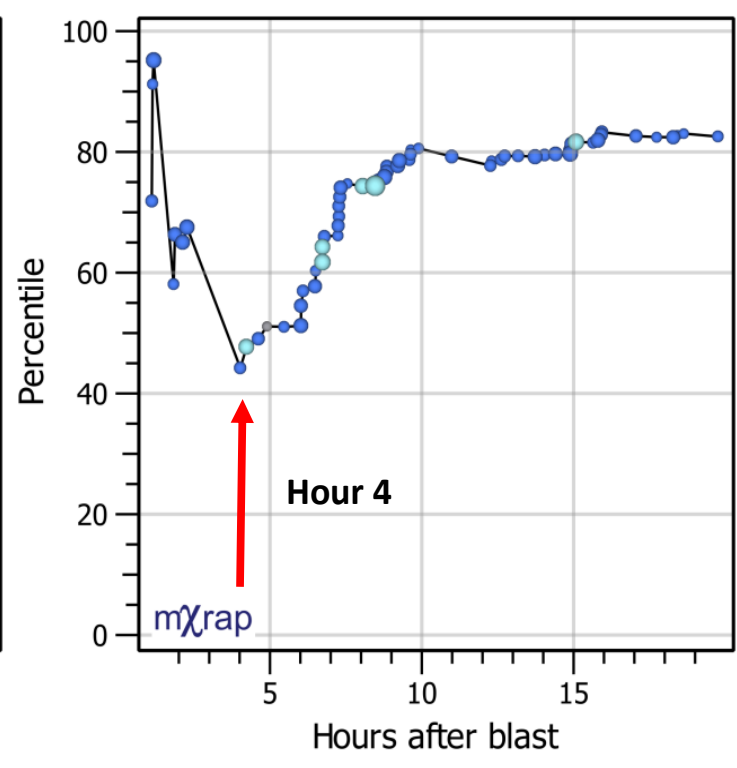

(b)

Figure 2 (a) Seismic response with envelopes; and, (b) seismic response expressed as percentage of the envelopes. The black line with the coloured spheres indicates the response curve

The second challenge was the fluctuation seen for the response in the percentile-time space. That is, there is high variation in the response percentile values at the start of the envelopes, and then a low variation at the end of the envelopes. At the start all of the MOL envelopes are at the exact same value and only after some time do they sufficiently separate and, hence, movement of the response in this time frame is exaggerated. In contrast, the ends of the MOL envelopes get more separated, which results in the response movement stagnating. In an attempt to reduce this fluctuation, movement is only considered 30 minutes after the envelopes start. This meant that all re-entry times had a minimum re-entry time of 90 minutes and a maximum re-entry time of 23 hours.

\subsection{Time of maximum curvature (2nd protocol)}

\subsubsection{V\&M method}

The 2nd protocol proposed for determining when conditions are favourable for re-entry is based on the time of maximum curvature $\left(\mathrm{T}_{\mathrm{MC}}\right)$. The $\mathrm{T}_{\mathrm{MC}}$ is the time at which the response transitions from a high to a low event rate when obeying the MOL. Vallejos and McKinnon (2010) defines $\mathrm{T}_{\mathrm{MC}}$ as:

$$
\mathrm{T}_{\mathrm{MC}}=\left[\mathrm{Kp} \sqrt{\frac{2 \mathrm{p}+1}{\mathrm{p}+2}}\right]^{\frac{1}{\mathrm{p}+1}}-\mathrm{c}
$$

Where parameters $\mathrm{K}, \mathrm{p}$ and $\mathrm{c}$ have the same definition as in Equation (1).

The $\mathrm{T}_{\mathrm{MC}}$ boundary is determined via a Monte Carlo simulation of the distributions of the MOL parameters. The same responses (blue and red lines) as in Figure 1 are shown in Figure 3, with the addition of the maximum $\mathrm{T}_{\mathrm{MC}}$ boundary as the grey line. The $\mathrm{V} \& \mathrm{M}$ method defines re-entry as favourable when the response curve moves through the $\mathrm{T}_{\mathrm{MC}}$ boundary line, which was assumed as the upper boundary of the scatter plot obtained from the Monte Carlo analysis. The response curve in this instance indicates the number of events in a two hour moving window. 


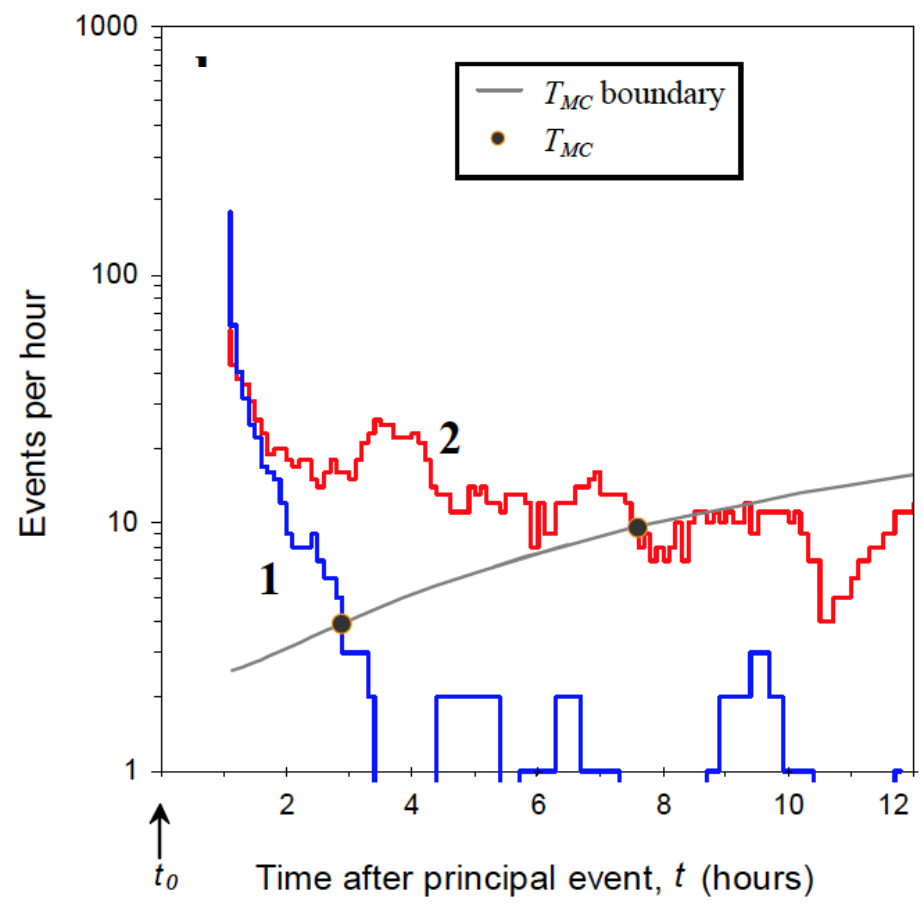

Figure 3 Event rate diagram for two seismic paths (Vallejos \& McKinnon 2010)

\subsubsection{Automated V\&M method}

In the automated V\&M method, the $\mathrm{T}_{\mathrm{MC}}$ boundary line is redefined in such a way that the K-parameter is eliminated. This then removes the need for a Monte Carlo simulation, and reduces computer processing time. The boundary is now expressed as the percentile of the $p$-parameter from the statistics:

$$
\mathrm{n}\left(\mathrm{T}_{\mathrm{MC}}\right)=\frac{\mathrm{T}_{\mathrm{MC}} \mathrm{p}}{\mathrm{p} \cdot \sqrt{\frac{2 \mathrm{p}+1}{\mathrm{p}+2}}}
$$

Where the parameters have the same definition as in Equation (1). Similar to the V\&M method, the conditions are taken to be favourable for re-entry when the response curve moves through the $\mathrm{T}_{\mathrm{MC}}$ boundary line. For this paper the $\mathrm{T}_{\mathrm{MC}}$ boundary is based on the $99.9 \%$ of the $\mathrm{p}$-parameter. The automated $V \& M$ method also uses a two hour moving time window to determine the response curve.

\subsection{Background level (3rd protocol)}

\subsubsection{V\&M method}

The third protocol is based on the background level of seismicity experienced on a site. Typically, most of the events recorded occur in the hours immediately after blasting; a typical diurnal chart is shown in Figure 4(a). The background level in the V\&M method was determined based on a Chi-squared test for the cumulative ascending distribution (CAD) of the diurnal charts, Figure 4(b). Vallejos and McKinnon (2010) argues that, evaluating the data in this way removes most of the events associated with blasting, and the events left in the other hour bins are indicative of a background level. The background level $B$ is then determined through:

$$
B=A_{T \%} \cdot A / T
$$

where:

$A_{T \%}$ is the percentage of background activity.

$A$ is the total activity for the time period of interest.

$T$ is the total non-omitted time bins of activity. 
Conditions are considered favourable when the response curve (based on a two hour moving window) reaches this background level.
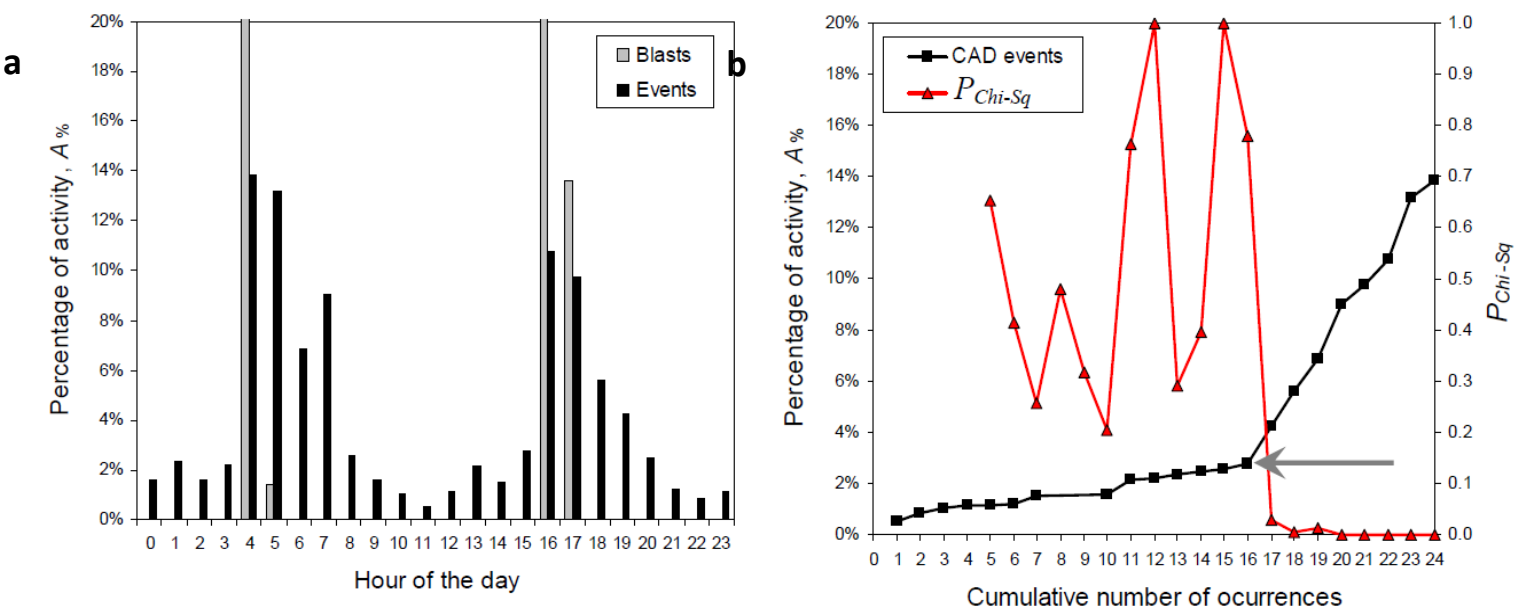

Figure 4 (a) Diurnal chart for a zone of a mine. (b) Cumulative ascending distribution of the hourly bins and the observed level of significance of the Chi-Square test ( $\left.P_{\text {chi-sq }}\right)$ for testing the hypothesis that the data set can be described by a uniform distribution (Vallejos \& McKinnon 2010)

\subsubsection{Automated V\&M method}

Vallejos and McKinnon (2010) does not explicitly state the distance and time window considered in their analyses. Nor do they make it clear if the same distance and time is considered for each response. However, to ensure an automated V\&M method, these two parameters need to be explicitly stated. These values may differ between mines, but should be consistently used within the areas investigated.

Determining the quasi-static background level is inherently difficult and not even the complex technique used by the V\&M method is likely to ensure consistency. Therefore, the technique used here aims to be more easily understood and implemented without having a large effect on the consistency of the protocol. The method used for the automated V\&M method also considers the CAD of the diurnal chart, but instead of finding a flex point in the data based on the $P_{\text {chi-sq }}$ value, it uses the 12 th highest bin.

\section{Case studies}

\subsection{Methodology}

Two open stope mines were considered for this study. The response statistics for both mine sites were based on the MOL fits for the blasts available at the time. All responses were limited to a radius of $150 \mathrm{~m}$ from the blast and an analysis time of 23 hours with an appropriate $M_{\text {min }}$-value. Only responses of 20 events or more are considered. This is mainly due to requiring this number of events, for fitting an $\mathrm{MOL}$ curve. In instances where data was too erratic, and the Anderson-Darling parameter (Anderson \& Darling 1954) indicated a poor fit, the curve was discarded.

For each of the mine sites, appropriate threshold values were determined for each of the protocols. These values were then consistently used to determine when conditions were favourable for re-entry. To determine the effectiveness of the methodology, a success is described as preventing exposure to a seismic event of local magnitude $\left(\mathrm{M}_{\mathrm{L}}\right)$ above a certain threshold. In this paper, two scenarios are considered, $\mathrm{M}_{\mathrm{L}}>0$ and $M_{L}>1$. Re-entry in this setup is defined as the earliest time when the thresholds for the three protocols are exceeded at the same time. 


\subsection{Results}

One of the two sites considered is Tasmania Mine. For this mine site a $\mathrm{M}_{\min }$ of -1.5 was used and $82 \mathrm{MOL}$ fits were possible from the database. Table 1 summarises of the parameters from the responses.

Table 1 Summary of the MOL parameter statistics for Tasmania Mine

\begin{tabular}{llll}
\hline Statistic & $\boldsymbol{p}$-value & $\boldsymbol{K}$-value & $\boldsymbol{c}$-value \\
\hline Mean & 0.67 & 8.22 & 0.00 \\
\hline Std. Dev. & 0.14 & 7.64 & 0.01 \\
Median & 0.67 & 8.22 & 0.00 \\
\hline Low-high & $0.31-1.01$ & $2.00-51.32$ & $0.00-0.03$ \\
\hline
\end{tabular}

Based on the automated V\&M method, re-entry occurs at the time when the response drops $3 \%$ in the envelope chart (1st protocol), it moves below the $99.9 \% \mathrm{~T}_{\mathrm{MC}}$ boundary line and it drops to below two times the background ratio. The background ratio, in this instance, is based on the last six months of data for a $500 \mathrm{~m}$ distance around the blast under consideration; the 12th highest event rate bin is used for the quasi-static background level.

As was discussed in the previous section; when none of the protocol thresholds were triggered, re-entry is taken as 23 hours. Also, the first 90 minutes are excluded to ensure the percentiles are not erratic and trigger the thresholds incorrectly. Events which occur within the first 90 minutes are considered avoided. Prevention of exposing workers or equipment to the occurrence of a large seismic event is considered a success whilst it is considered a failure when workers or equipment was exposed to the occurrence of the large event. Based on these thresholds the following results were obtained (Table 2):

Table 2 Summary of the re-entry results for Tasmania Mine

\begin{tabular}{lll}
\hline Details & Automated V\&M method & 'Blanket' rule \\
\hline Average re-entry time (hours) & 7.7 & 7.7 \\
\hline \% success & $\mathbf{M}_{\mathrm{L}}>\mathbf{1}$ & \\
\hline \% failure & $84 \%$ & $89 \%$ \\
& $16 \%$ & $11 \%$ \\
\hline \% success & $\mathbf{M}_{\mathrm{L}}>\mathbf{0}$ & \\
\hline \% failure & $68 \%$ & $72 \%$ \\
\hline
\end{tabular}

The performance of the method is reasonable. For all events of $M_{L}>1$ that occurred in a blasting sequence within 23 hours, $84 \%$ of them were excluded with the automated V\&M method. However, using a 'blanket' rule of 7.7 hours, one is able to improve the number of prevented events with an additional $5 \%$. For events of $\mathrm{M}_{\mathrm{L}}>0$ these values are similar, and one needs to conclude that the automated V\&M method, as described in this paper, is no better than the 'blanket' rule method for the same amount of hours.

A similar study was performed on Mine A. This site has large events pre-dominantly on large geological structures. The MOL parameters are based on 135 responses, and a $\mathrm{M}_{\min }-2.0$ was used for this analysis. Table 3 summarises the MOL parameters used for the analysis. 
Table 3 Summary of the MOL parameter statistics for Mine A

\begin{tabular}{llll}
\hline Statistic & $\boldsymbol{p}$-value & $\boldsymbol{K}$-value & $\boldsymbol{c}$-value \\
\hline Mean & 0.77 & 9.32 & 0.00 \\
Std. Dev. & 0.21 & 11.51 & 0.01 \\
Median & 0.77 & 9.32 & 0.00 \\
Low-high & $0.26-1.57$ & $2.12-87.74$ & $0.00-0.03$ \\
\hline
\end{tabular}

Table 4 summarises the results obtained for Mine A. For this mine site the automated V\&M method, outperforms the 'blanket' rule for the same average exclusion time. For $M_{L}>2$ the two methods have the same performance but for $M_{L}>1$ the automated V\&M method out performs the 'blanket' rule by $7 \%$ and for $M_{L}>0$ this increases to $11 \%$. These are significant gains in the number of events prevented, and indicate that this methodology might be helpful at some underground mines.

Table 4 Summary of the analysis results for Mine A

\begin{tabular}{lll}
\hline Details & Automated V\&M method & 'Blanket' rule \\
\hline Average re-entry time (hours) & 10.4 & 10.4 \\
\hline \% success & $\mathbf{M}_{\mathbf{L}}>\mathbf{2}$ & \\
\hline \% failure & $73 \%$ & $73 \%$ \\
\hline \% success & $27 \%$ & $27 \%$ \\
\hline \% failure & $\mathbf{M}_{\mathbf{L}}>\mathbf{1}$ & \\
\hline & $69 \%$ & $62 \%$ \\
\hline \% success & $31 \%$ & $38 \%$ \\
\hline \% failure & $\mathbf{M}_{\mathbf{L}}>0$ & \\
\hline
\end{tabular}

\section{Discussion}

The V\&M method has been automated and successfully implemented on two seismic sites. It was shown that, for Tasmania Mine the automated V\&M method was less effective than applying a 'blanket' rule for the same time. This would indicate the method does not improve on current re-entry practices. On the other hand, the automated V\&M method showed a moderate improvement for Mine A. This would suggest there are some scenarios where this method would outperform a typical 'blanket' rule. However, the methodology needs to be systematically applied to improve the quantification of the method reliability and to determine and under which conditions the method performs best.

By using the thresholds, one is able to define re-entry as a set of rules. This is useful as the experience is captured through the back analysis process and then followed by the personnel involved. It reduces the influence and motivational bias of the onsite engineer making the decision.

From this work, there are a few areas where further development may greatly improve the method and consistency of the automated V\&M method. The suggestions for improvement are:

- Instead of manually finding and fitting responses with the MOL, use the methodology proposed by Woodward et al. (2017) to search and fit all available responses. 
- The system sensitivity for micro-seismic systems differs spatially depending on the local density of the sensors. To try and eliminate this effect, a $M_{\min }$ was used to filter the data. Since the sensitivity differs spatially further improvement is possible with adjustment to the K-parameter (productivity) according to the local sensitivity.

- The fluctuations seen during the analysis process of the first protocol needs to be investigated further. This would ensure more consistent results.

\section{Conclusion}

The V\&M method was codified to enable automation for practical implementation on mine sites with a comprehensive seismic data record. It was shown the methodology, in some cases, may be an improvement on the widely used 'blanket' rule implemented on many mine sites.

For the Tasmania Mine the 'blanket' rule was $~ 5 \%$ better at preventing exposure to large events. For Mine $A$ the automated V\&M method was $\sim 10 \%$ better than the 'blanket' rule. The condition under which the method is more accurate for re-entry is not clear and requires further investigation. There are also some indications and suggestions made in this paper for the improvement of the implementation.

\section{Acknowledgement}

We acknowledge the support of the mXrap Consortium and the Australian Centre for Geomechanics during this project.

\section{References}

Anderson, TW \& Darling, DA 1954, 'A test of goodness of fit', Journal of the American Statistical Association, vol. 49, no. 268, pp. $765-769$.

Harris, PC \& Wesseloo, J 2015, mXrap, version 5, Australian Centre for Geomechanics, Perth, www.mXrap.com

Hudyma, M 2008, Analysis and Interpretation of Clusters of Seismic Events in Mines, PhD thesis, The University of Western Australia, Perth.

Mendecki, A 'Forecasting seismic hazard in mines', in Y Potvin, J Carter, A Dyskin \& R Jeffrey (eds), Proceedings of the 1st Southern Hemisphere International Rock Mechanics Symposium, 16-19 September 2008, Perth, Australian Centre for Geomechanics, Perth, pp. 55-69.

Potvin, Y 2009, 'Strategies and tactics to control seismic risks in mines', Journal of the Southern African Institute of Mining and Metallurgy, vol. 109, pp. 177-186.

Utsu, T 1961, 'A statistical study on the occurrence of aftershocks', Geophysical Magazine, vol. 30, no. 4, pp. 521-605.

Vallejos, JA \& McKinnon, SD 2010, 'Temporal evolution of aftershock sequences for re-entry protocol development in seismically active mines', in M Van Sint Jan \& Y Potvin (eds), Proceedings of the Fifth International Seminar on Deep and High Stress Mining, 6-8 October 2010, Santiago, Australian Centre for Geomechanics, Perth, pp. 199-214.

Woodward, K, Morkel, IG \& Wesseloo, J 2015, mXrap Software App, Mining Induced Seismicity - Omori Analysis Tools, version 1, Australian Centre for Geomechanics, Perth, www.mXrap.com

Woodward, K, Wesseloo, J \& Potvin, Y 2017, 'The spatial and temporal assessment of clustered and time-dependent seismic responses to mining', in J Wesseloo (ed.), Proceedings of the Eighth International Conference on Deep and High Stress Mining, 28-30 March 2017, Perth, Australian Centre for Geomechanics, Perth, in press. 
